\title{
Novedades de las Guías de la Sociedad Europea de Cardiología 2020 sobre manejo del síndrome coronario agudo sin elevación persistente del segmento ST
}

\author{
Dres. Carlos Guamán, Gimena Loza, Juan Albistur
}

\section{Resumen}

Las enfermedades cardiovasculares representan la primera causa de muerte en el mundo. El manejo de los síndromes coronarios ha avanzado formidablemente en los últimos 50 años, reduciendo el riesgo isquémico, a expensas del consiguiente aumento del riesgo hemorrágico. La Sociedad Europea de Cardiología publicó en el año 2020 la guía sobre el manejo de síndrome coronario agudo sin elevación del segmento ST, donde se destacan cambios en los algoritmos de estratificación de riesgo y la terapia antiplaquetaria y anticoagulante como dos de los aspectos principales. En el presente editorial se resumen las principales novedades publicadas en este documento.

Palabras clave: $\quad$ SÍNDROME CORONARIO AGUDO; INFARTO DE MIOCARDIO; ANTIAGREGACIÓN PLAQUETARIA; INTERVENCIÓN CORONARIA PERCUTÁNEA

News from the European Society of Cardiology 2020 Guidelines on the Management of Acute Coronary Syndrome without Persistent ST-Segment Elevation

\section{Summary}

Cardiovascular disease is the leading cause of death worldwide. The management of coronary syndromes has advanced dramatically in the last 50 years, reducing ischemic risk, but observing an increase in bleeding risk. The European Society of Cardiology published in 2020 the guideline on the management of acute coronary syndrome without ST-segment elevation, where changes in risk stratification algorithms and antiplatelet and anticoagulant therapy are highlighted as two of the main aspects. This editorial summarizes the main developments published in this document.

Key - words: $\quad$ ACUTE CORONARY SYNDROME; MYOCARDIAL INFARCTION; ANTI-PLATELET AGGREGATION; PERCUTANEOUS CORONARY INTERVENTION

Novidades da Sociedade Europeia de Cardiologia 2020 Diretrizes sobre o Tratamento da Síndrome Coronariana Aguda sem Elevação Persistente do Segmento ST

\section{Resumo}

A doença cardiovascular é a principal causa de morte em todo o mundo. O manejo das síndromes coronarianas avançou dramaticamente nos últimos 50 anos, reduzindo o risco isquêmico, mas observando um aumento no risco de sangramento. A Sociedade Europeia de Cardiologia publicou em 2020 a diretriz sobre o manejo da síndrome coronariana aguda sem supradesnivelamento do segmento ST, onde as alterações nos algoritmos de estratificação de risco e na terapia antiplaquetária e anticoagulante são destacadas como dois dos principais aspectos. Este editorial resume os principais desenvolvimentos publicados neste documento.

Palavras chave: SÍNDROME CORONÁRIA AGUDA; INFARTO DO MIOCÁRDIO AGREGAÇÃO ANTIPLATELET; INTERVENÇÃO CORONÁRIA PERCUTÂNEA

Centro Cardiovascular Universitario, Hospital de Clínicas. Montevideo, Uruguay.

Correspondencia: Dr. Carlos Guamán. Correo electrónico: cgv0792@gmail.com

Los autores declaran no tener conflictos de intereses.

Recibido Feb 1, 2021; aceptado Feb 23, 2021 


\section{Introducción}

Las enfermedades cardiovasculares continúan siendo la principal causa de muerte en el mundo occidental y específicamente en nuestro país. La enfermedad coronaria ateroesclerótica es una de las más prevalentes y con mayor mortalidad, pudiéndose presentar como síndromes coronarios crónicos o $\operatorname{agudos}^{(1)}$. Los síndromes coronarios agudos sin elevación persistente del segmento ST (SCASEST) se han convertido en la forma de presentación más frecuente $^{(2)}$. En octubre de 2020, la Sociedad Europea de Cardiología publicó una nueva guía sobre el manejo de los SCASEST ${ }^{(3)}$. A continuación, se realiza un resumen de las principales novedades.

\section{Diagnóstico}

Se continúa resaltando la importancia del correcto diagnóstico clínico e interpretación electrocardiográfica y aporta novedades en cuanto a la utilidad de los biomarcadores.

El uso de troponinas cardíacas de alta sensibilidad permite disminuir el tiempo de extracción de la segunda muestra, reduciendo sustancialmente el retraso diagnóstico, el tiempo de traslado del paciente a sala de hemodinamia y el costo del sistema de salud. A su vez, identifica candidatos adecuados para el alta temprana y el manejo ambulatorio.

Se recomienda un esquema de rápida confirmación o descarte (rule-in/rule-out) basado en la dosificación a la 0 y 1 hora, o en segundo lugar a las 0 y 2 horas, sustituyendo al recomendado previamente de 0 y 3 horas. Debe considerarse no sólo el valor absoluto, sino también la variación entre ambas dosificaciones para descartar o confirmar. Estos esquemas son seguros y su sensibilidad es mayor a $99 \%$, incluso en pacientes que se presentan con menos de dos horas de iniciado los síntomas. En aquellos que se presentan con menos de una hora de iniciado los síntomas, podría ser necesario extraer una tercera muestra a las 3 horas. Por el contrario, si el inicio de los síntomas es mayor a 3 horas, un único valor de troponinas a la hora 0 es suficiente. No se recomiendan otros biomarcadores adicionales a las troponinas de alta sensibilidad con fines diagnósticos.

El segundo aspecto relevante del capítulo diagnóstico es el rol de la angiotomografía. En pacientes con probabilidad pretest de enfermedad coronaria baja-intermedia, y con electrocardiograma (ECG) y troponinas normales o no concluyentes, la realización de una angiotomografía coronaria es actualmente una indicación clase I, nivel de evidencia A, para excluir el SCASEST.

\section{Estratificación de riesgo}

En este aspecto existen dos puntos novedosos a destacar. Se incorpora la medición de péptidos natriuréticos como indicación clase IIa, nivel de evidencia $\mathrm{B}$, con objetivo pronóstico como complemento a la medición sistemática de las troponinas. En segundo lugar, se baja el grado de recomendación para el uso del score de GRACE a clase IIa, nivel de evidencia B.

\section{Tratamiento antitrombótico}

La doble antiagregación en base a ácido acetilsalicílico (AAS) y un inhibidor de P2Y12 continúa siendo indicación IA, con preferencia de los inhibidores P2Y12 más potentes, como prasugrel o ticagrelor. Sin embargo, basado en el estudio ISAR-REACT $5^{(4)}$, con nivel de evidencia IIa, se recomienda la utilización de prasugrel sobre ticagrelor para pacientes que van a realizarse angioplastia coronaria.

Uno de los aspectos de mayor relevancia y que determina un cambio en la práctica clínica habitual se refiere al pretratamiento con inhibidores de la P2Y12. Según la evidencia disponible el pretratamiento no mejora los resultados isquémicos, y sí aumenta de forma significativa las complicaciones hemorrágicas, por lo que no se recomienda el uso rutinario de un inhibidor del receptor de P2Y12 en pacientes con SCASEST en quienes la anatomía coronaria aún no es conocida y se ha planificado una estrategia invasiva temprana (recomendación clase III, nivel A). Para los pacientes en los que no se plantea una estrategia invasiva temprana, podría considerarse el pretratamiento con un inhibidor del receptor $\mathrm{P} 2 \mathrm{Y} 12$ si el riesgo de sangrado no es alto (recomendación clase IIb, nivel C).

La anticoagulación sigue siendo una terapéutica fundamental tanto en el preprocedimiento como durante el procedimiento. En cuanto al tratamiento anticoagulante, durante el procedimiento intervencionista se ha bajado el grado de recomendación de la bivalirudina a clase IIb, nivel de evidencia A, como alternativa a la heparina no fraccionada.

\section{Tratamiento posintervención y mantenimiento}

La doble antiagregación plaquetaria por 12 meses continúa siendo recomendada, independientemente del tipo de stent (recomendación clase I, nivel A), siempre que no existan contraindicaciones y pudiendo individualizar la duración según el escenario clínico. El balance entre el riesgo isquémico y el hemorrágico debe incluir características del paciente, forma de presentación, comorbilidades, necesidad de medicación concomitante y aspectos relacionados al procedimiento coronario (figura 1). 


\begin{tabular}{|c|c|}
\hline A. Riesgo hemorrágico & B. Riesgo trombótico \\
\hline CRITERIO MAYOR & RIESGO TROMBÓTICO ALTO \\
\hline - Uso previo de ACO & Enfermedad coronaria compleja y al menos 1 criterio \\
\hline - ERC con FG estimado $<30 \mathrm{~m} / / \mathrm{min} / 1,73 \mathrm{~m}^{2}$ & POTENCIADORES DE RIESGO \\
\hline - $\mathrm{Hb}<11 \mathrm{~g} / \mathrm{dL}$ & - Diabetes mellitus que requiere medicación \\
\hline - Sangrado espontáneo que requirió hospitalización y/o transfusión en los & - Historia de infarto de miocardio recurrente \\
\hline últimos 6 meses o en cualquier momento, si es recurrente & - Enfermedad coronaria multivaso \\
\hline - Trombocitopenia $<100.000 / \mathrm{mL}$ previo a la ICP & - Enfermedad polivascular (enfermedad coronaria + EAP) \\
\hline - Diátesis hemorrágica & - Enfermedad coronaria prematura ( $<45$ años) o acelerada (nueva lesión en \\
\hline - Cirrosis hepática con hipertensión portal & un periodo de 2 años) \\
\hline - Cáncer activo en los últimos 12 meses (excluyendo cáncer de piel no - & - Enfermedad inflamatoria sistémica concomitante (VIH, LES, artritis crónica) \\
\hline melanoma) & $\begin{array}{l}\text { - ERC con FG estimado entre } 15-59 \mathrm{ml} / \mathrm{min} / 1,73 \mathrm{~m}^{2} \\
\text { ASPECTOS TÉCNICOS }\end{array}$ \\
\hline - Hemorragia intracraneal espontánea previa & - Al menos 3 stent implantados \\
\hline - Hemorragia intracraneal traumática en los últimos 12 meses & - Al menos 3 lesiones tratadas \\
\hline - Malformación arteriovenosa cerebral & - Longitud total de stent $>60 \mathrm{~mm}$ \\
\hline - ACV isquémico moderado o severo (NIHSS>5) en los últimos 6 meses & - Historia de revascularización compleja ( $\mathrm{TCl}$, bifurcación con $\geq 2$ stent \\
\hline - Cirugía o trauma mayor en los 30 dias previo a la ICP & implantados, oclusión total crónica, stent en último vaso permeable) \\
\hline $\begin{array}{l}\text { - Cirugia mayor no diferible bajo DAPT } \\
\text { CRITERIO MENOR }\end{array}$ & - Historia de trombosis de stent bajo tratamiento antiplaquetario \\
\hline - Edad $\geq 75$ años & \\
\hline - ERC con FG estimado entre $30-59 \mathrm{~m} / / \mathrm{min} / 1,73 \mathrm{~m}^{2}$ & \\
\hline - Hb $11-12,9 \mathrm{~g} / \mathrm{dL}$ en hombres o $11-11.9 \mathrm{~g} / \mathrm{dL}$ en mujeres & \\
\hline $\begin{array}{l}\text { - Sangrado espontáneo que requirió hospitalización y/o transfusión en los } \\
\text { últimos } 12 \text { meses que no cumple criterio mayor }\end{array}$ & \\
\hline - Uso crónico de antiinflamatorios no esteroideos o corticoides & \\
\hline - Cualquier ACV en cualquier momento que no cumple criterio mayor & \\
\hline
\end{tabular}

Figura 1. A: criterios de riesgo hemorrágico basados en la Academic Research Consortium for High Bleeding Risk. B: criterios de alto riesgo isquémico para definir tratamiento extendido con segundo antitrombótico. ACO: anticoagulante oral; ACV: ataque cerebrovascular; DAPT: doble antiagregación plaquetaria; ERC: enfermedad renal crónica; FG: filtrado glomerular; Hb: hemoglobina; ICP: intervención coronaria percutánea; LES: lupus eritematoso sistémico; NIHSS: National Institute of Health Stroke Scale; TCI: tronco de la arteria coronaria izquierda; VIH: virus de inmunodeficiencia humana. Modificado de las tablas 7 y 11 de la Guía de la Sociedad Europea de Cardiología 2020 sobre el manejo de SCASEST.

Se introduce un nuevo score acorde a la Academic Research Consortium for High Bleeding Risk (ARC-HBR) (figura 1A) para evaluar riesgo de sangrado al momento de la intervención coronaria percutánea (ICP). Éste establece criterios mayores y menores y considera alto riesgo cuando se presentan al menos un criterio mayor o dos criterios menores. La evaluación del riesgo isquémico continúa siendo fundamental, establecida de forma clara (figura 1B), y permite definir la duración óptima de la doble terapia antiagregante.

Por lo tanto, en base a este análisis, se establece que la duración de la doble terapia antiagregante, luego del implante de un stent, podría ir desde un mes en pacientes con muy alto riesgo de sangrado y tres meses en aquellos con alto riesgo de sangrado, hasta más de un año en aquellos con predominio del riesgo isquémico (figura 2). La prolongación de la doble terapia antiagregante más allá del año en pacientes con alto riesgo de eventos isquémicos y sin riesgo incrementado de sangrado se recomienda actualmente con una indicación de clase IIa, nivel de evidencia $\mathrm{A}$, lo que hace que debamos considerar esta estrategia cada vez con más frecuencia.

\section{Pacientes con fibrilación auricular sin válvula protésica ni estenosis mitral moderada-severa y SCASEST}

Se presentan aquí tres escenarios clínicos: pacientes que se realizarán ICP, los que reciben exclusivamente tratamiento médico y aquellos en que se plantea realizar cirugía de revascularización miocárdica. Debemos valorar cuidadosamente el manejo antitrombótico de quienes tengan indicación de anticoagulación por la fibrilación auricular (FA) $\left(\mathrm{CHA}_{2} \mathrm{DS}_{2}-\mathrm{VASC} \geq 1\right.$ en hombres $y \geq 2$ en mujeres), un grupo de difícil manejo en la práctica habitual por la acumulación de terapia antiagregante $\mathrm{y}$ anticoagulante.

\section{Pacientes que se someterán a intervención coronaria percutánea}

Sin duda, este es uno de los aspectos centrales de la nueva guía, que probablemente nos obliga a repensar el manejo habitual de estos pacientes. Un porcentaje de pacientes no despreciable que se someten a ICP tiene indicación de anticoagulación oral por presentar FA; múltiples estudios randomizados han intentado responder cuál es la mejor estrategia de tratamiento antitrombótico en estos casos, y la 


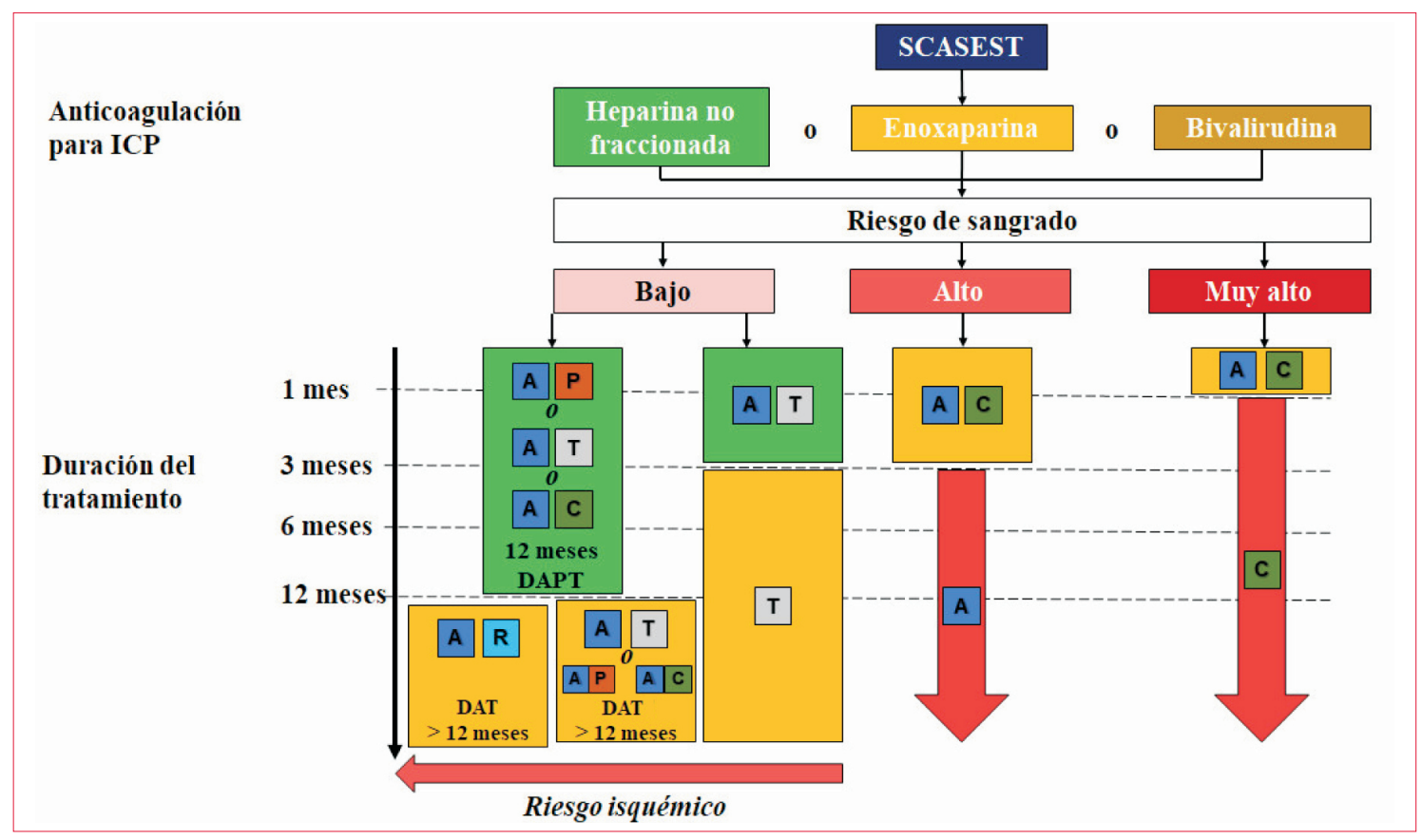

Figura 2. Algoritmo de tratamiento antitrombótico en pacientes sin fibrilación auricular (FA) que van a intervención coronaria percutánea en SCASEST. A: ácido acetilsalicílico; C: clopidogrel; P: prasugrel; R: rivaroxabán; T: ticagrelor. DAT: doble terapia antiagregante. En verde se representan las indicaciones clase I y en amarillo las indicaciones clase II. Modificado de figura 7 de la Guía de la Sociedad Europea de Cardiología 2020 sobre el manejo de SCASEST.

guía propone una nueva solución a esta encrucijada (figura 3). Se prefiere los anticoagulantes directos sobre los antagonistas de la vitamina $\mathrm{K}$, y se establece con un grado de recomendación de clase I, nivel de evidencia A, que la estrategia de elección consista en realizar triple terapia solamente durante el período de tiempo de internación (hasta una semana), continuando luego con doble terapia en base a un anticoagulante directo con un solo antiagregante, preferiblemente clopidogrel. En el caso de requerir antagonistas de la vitamina $\mathrm{K}$ (por ejemplo, pacientes con válvulas mecánicas), se mantiene la misma estrategia temporal. Esta estrategia puede modificarse según el riesgo de sangrado, haciendo más breve la duración de la doble terapia y priorizando la terapia solo con anticoagulación, o según el riesgo isquémico, en cuyo caso podría prolongarse la triple terapia hasta completar un mes. Esto constituye un cambio importante respecto a las recomendaciones previas que no consideraban la suspensión de la doble antiagregación en ningún paciente que tuviera menos de un mes desde el procedimiento.

Es destacable que en pacientes con alto riesgo de trombosis del stent, independientemente del tipo, la doble terapia con anticoagulación oral y ticagrelor o prasugrel, pueda ser considerada como una alternativa a la triple terapia con indicación clase IIb, nivel de evidencia C. En pacientes seleccionados con alto riesgo isquémico se puede considerar la anticoagulación oral más antiagregación plaquetaria simple extendida más allá del primer año, con indicación clase IIa, nivel de evidencia C.

\section{Pacientes que se manejarán con tratamiento médico}

Si bien también es reconocido el beneficio de la doble antiagregación en estos pacientes, no existe preocupación por la trombosis del stent, por lo que el escenario es menos complejo. Se plantea con un nivel de indicación clase IIa, nivel de evidencia C, mantener hasta el año del procedimiento un antiagregante adicional al tratamiento anticoagulante.

\section{Pacientes que se someterán a cirugía de revascularización miocárdica}

Debe evitarse la triple terapia por el alto riesgo de sangrado que implica el posoperatorio de cirugía cardíaca, y se resalta la estrategia de reinstalar la anticoagulación oral tan pronto como sea posible en aquellos pacientes que tienen una indicación establecida, en combinación con un antiagregante.

\section{Tratamiento invasivo}

En este aspecto no existen cambios significativos con respecto a las recomendaciones de la guía anterior. Se establecen plazos para una estrategia invasiva, de acuerdo a la estratificación de riesgo de eventos isquémicos agudos a corto plazo, conside- 


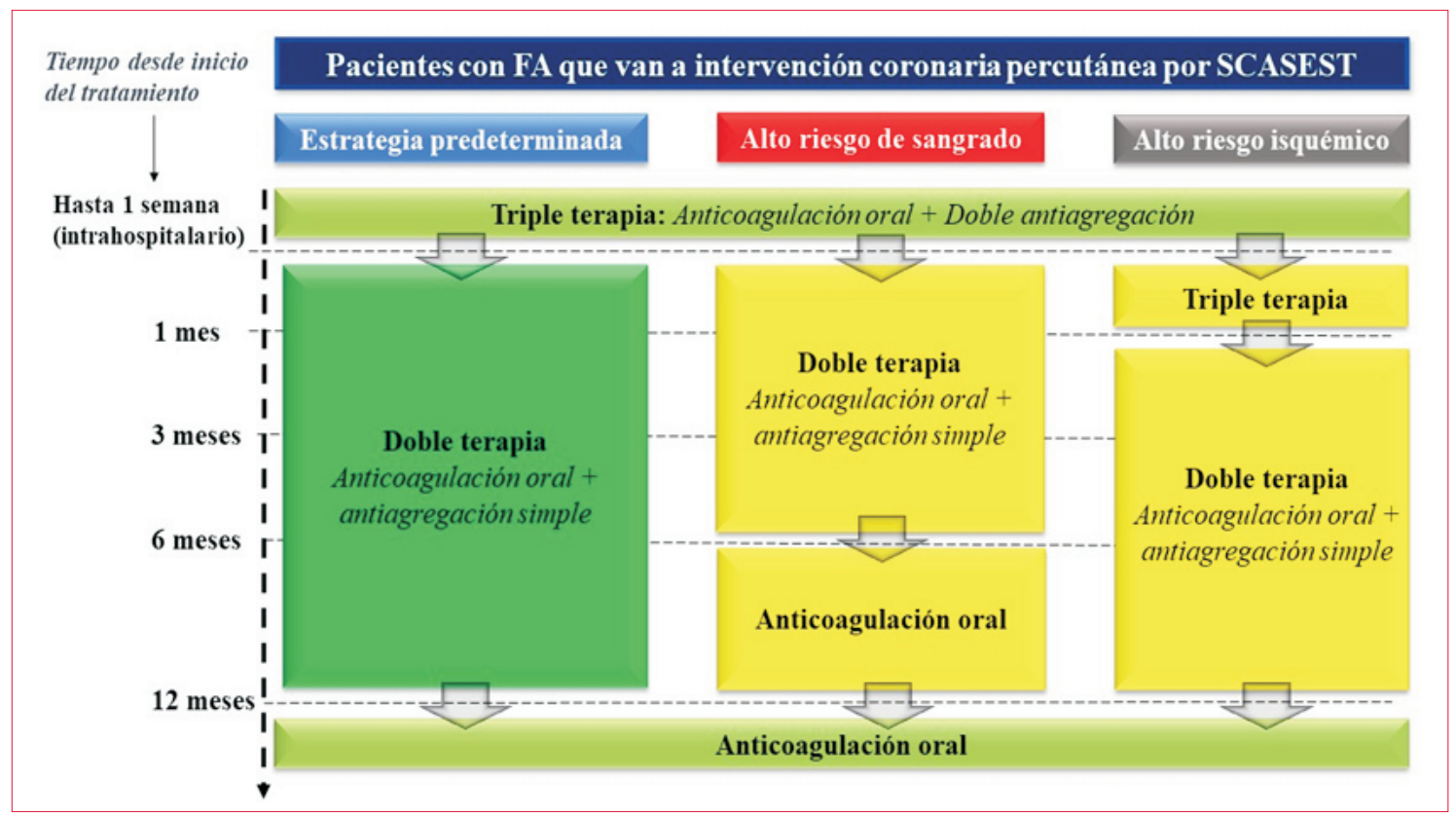

Figura 3. Algoritmo de tratamiento antitrombótico en pacientes con fibrilación auricular que van a intervención coronaria percutánea en SCASEST. En verde oscuro se representan las indicaciones clase I y en amarillo las indicaciones clase II. Modificado de figura 8 de la Guía de la Sociedad Europea de Cardiología 2020 sobre el manejo de SCASEST.

rando criterios clínicos, electrocardiográficos y de biomarcadores. Se plantea una estrategia invasiva inmediata (menos de 2 horas) para pacientes de riesgo muy alto, y una estrategia invasiva temprana (menos de 24 horas) para los de riesgo alto (figura 4); en aquellos pacientes que no cumplen con ninguno de estos criterios y se clasifican como de riesgo bajo, deberá individualizarse el manejo con una estrategia invasiva selectiva.

Un aspecto siempre controvertido se refiere a pacientes con paro cardíaco resucitado extrahospitalario que se encuentran hemodinámicamente estables y sin supradesnivel del segmento ST en el ECG. En este sentido, la estrategia de cineangiocoronariografía (CACG) inmediata rutinaria no ha demostrado ser superior a una estrategia retrasada, por lo cual se recomienda con un grado de recomendación IIa, nivel de evidencia $\mathrm{B}$, una estrategia de CACG retrasada versus inmediata.

\section{Infarto de miocardio sin enfermedad coronaria obstructiva}

Se crea una nueva sección que aborda el manejo del infarto agudo de miocardio sin enfermedad coronaria obstructiva (MINOCA, por su sigla en inglés, Myocardial infarction with non-obstructive coronary arteries). Se resalta el hecho de que las troponinas son órgano-específicas y no enfermedad-específicas, por lo que su elevación equivale a diagnóstico de daño miocárdico. Basándose en la cuarta definición de infarto, para establecer el diagnóstico de
MINOCA, primero se deben excluir otras causas de daño miocárdico: causas cardíacas no isquémicas (como el síndrome de takotsubo y la miocarditis) y causas extracardíacas (como tromboembolia pulmonar, anemia severa, sepsis). Una vez etiquetado como MINOCA, se inicia un algoritmo diagnóstico que incluye imagen intracoronaria, ecocardiografía transtorácica y resonancia cardíaca, entre otros ${ }^{(5)}$. Se destaca la importancia de la resonancia cardíaca para establecer el diagnóstico y se recomienda su realización en todos los pacientes sin una causa clara, con indicación clase I, nivel de evidencia B. En $8 \%$ a $25 \%$ de los casos la etiología permanece sin determinarse, recomendándose realizar el mismo tratamiento de prevención secundaria que en la enfermedad ateroesclerótica, con indicación clase IIb, nivel de evidencia $\mathrm{C}$.

\section{Disección coronaria espontánea}

Se dedica por primera vez una sección a esta entidad, que constituye hasta $4 \%$ de los casos de SCA en la población general (y hasta $35 \%$ en las mujeres menores de 60 años). La disección coronaria se define como la separación de las capas arteriales de causa no ateroesclerótica, traumática ni iatrogénica. Se reconocen factores de riesgo, dentro de los cuales se destacan el sexo femenino, el tratamiento hormonal, el embarazo, entre otros. En ocasiones la angiografía es muy sugestiva, pero en otros casos es necesario el uso de métodos de imagen intracoronario para confirmar el diagnóstico. Dado el alto grado de 


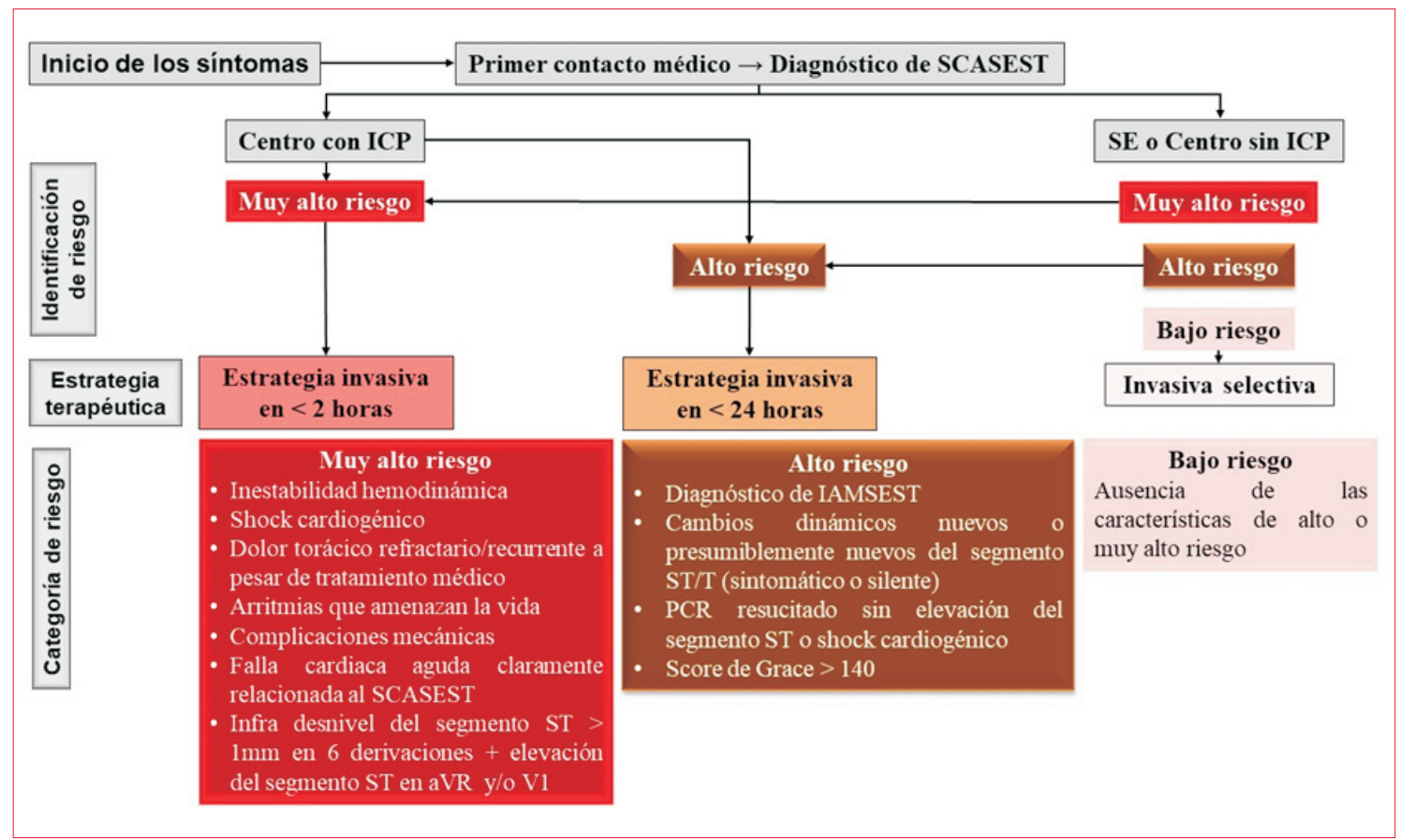

Figura 4. Algoritmo para la selección de la estrategia invasiva en SCASEST. IAMSEST: infarto agudo de miocardio sin elevación del segmento ST; PCR: paro cardiorrespiratorio; SE: servicio de emergencia. Modificado de figura 9 de la Guía de la Sociedad Europea de Cardiología 2020 sobre el manejo de SCASEST.

resolución espontánea, el tratamiento es prácticamente siempre médico, sin necesidad de revascularización, exceptuando casos con isquemia persistente. Según la evidencia actual, que es escasa, se reconoce la hipertensión arterial como un factor de riesgo de recurrencia, por lo cual el control intensivo de la presión arterial constituye un objetivo. El tratamiento con betabloqueantes ha demostrado beneficio en disminuir las recurrencias. Con respecto al tratamiento antitrombótico, existe evidencia controversial.

\section{Conclusiones}

Las Guías de la Sociedad Europea de Cardiología nos brindan información práctica, basada en la evidencia, especialmente en el tratamiento antiagregante, el cual ha experimentado grandes cambios basados en recientes ensayos clínicos randomizados, brindando algoritmos prácticos que buscan el equilibrio entre el riesgo isquémico y hemorrágico para seleccionar el tratamiento antiagregante y su duración óptima. Reafirman conceptos de estratificación de riesgo para optimizar la elección de la estrategia de manejo, y crean nuevas secciones sobre MINOCA y disección coronaria, poblaciones cuyo abordaje representa un reto en la práctica clínica.
Carlos Guamán, https://orcid.org/0000-0002-1065-1988 Gimena Loza, https://orcid.org/0000-0001-9555-7431 Juan Albistur, https://orcid.org/0000-0002-0282-5263

\section{Bibliografía}

1. Roger VL, Go AS, Lloyd-Jones DM, Benjamin EJ, Berry JD, Borden WB, et al; American Heart Association Statistics Committee and Stroke Statistics Subcommittee. Heart disease and stroke statistics--2012 update: a report from the American Heart Association. Circulation. 2012;125(1):e2-e220. doi: 10.1161/CIR.0b013e31823ac046 Fe de errata en: Circulation. 2012;125(22):e1002

2. Puymirat E, Simon T, Cayla G, Cottin Y, Elbaz M, Coste P, et al. Acute myocardial infarction: changes in patient characteristics, management, and 6-month outcomes over a period of 20 years in the FAST-MI Program (French Registry of Acute ST-Elevation or Non-ST-Elevation Myocardial Infarction) 1995 to 2015. Circulation. 2017;136(20):1908-1919. doi: 10.1161/CIRCULATIONAHA.117.030798

3. Collet JP, Thiele H, Barbato E, Barthélémy O, Bauersachs J, Bhatt DL, et al. 2020 ESC Guideli- 
nes for the management of acute coronary syndromes in patients presenting without persistent ST-segment elevation. Eur Heart J. 2020:ehaa575. doi: 10.1093/eurheartj/ehaa575

4. Mayer K, Bongiovanni D, Karschin V, Sibbing D, Angiolillo DJ, Schunkert H, et al. Ticagrelor or prasugrel for platelet inhibition in acute coronary syndrome patients: the ISAR-REACT 5 trial. J Am
Coll Cardiol. 2020;76(21):2569-2571. doi: 10.1016/j. jacc.2020.09.586

5. Thygesen K, Alpert JS, Jaffe AS, Chaitman BR, Bax JJ, Morrow DA, White HD. Fourth Universal Definition of Myocardial Infarction (2018). J Am Coll Cardiol. 2018;72(18):2231-64. doi: 10.1016/j.jacc.2018.08.1038 